\title{
Research Paper \\ Childhood Emotional Abuse and Borderline Personality Disorder Features: The Me- diating Roles of Attachment Style and Emotion Regulation
}

\author{
Hossein Pourshahriar ${ }^{1},{ }^{*}$ Hossein Alizade $^{2} \mathbb{C}^{\text {, Kazem Rajaeinia }}{ }^{3}$
}

\begin{abstract}
1. PhD in Psychology, Assistant Professor, Department of Applied Psychology, Faculty of Education and Psychology, Shahid Beheshti University, Tehran, Iran. 2. MSc., Department of Clinical \& Health Psychology, Faculty of Education and Psychology, Shahid Beheshti University, Tehran, Iran.

3. MSc., Department of Educational \& Development Psychology, Faculty of Education and Psychology, Shahid Beheshti University, Tehran, Iran
\end{abstract}

Received: 07 Feb 2017

Accepted: 09 May 2018

Key words: Attachment styles, Borderline personality disorder, Emotion regulation, Emotional abuse, Structural equation modeling ditation: Pourshahriar H, Alizade H, Rajaeinia K. Childhood Emotional Abuse and Borderline Personality Disorder Features: The Mediating Roles of Attachment Style and Emotion Regulation (Persian)]. Iranian Journal of Psychiatry an.d Clinical Psychology. 2018; 24(2):148-163. http://dx.doi.org/10.32598/ijpcp.24.2.148

http://dx.doi.org/10.32598/ijpcp.24.2.148

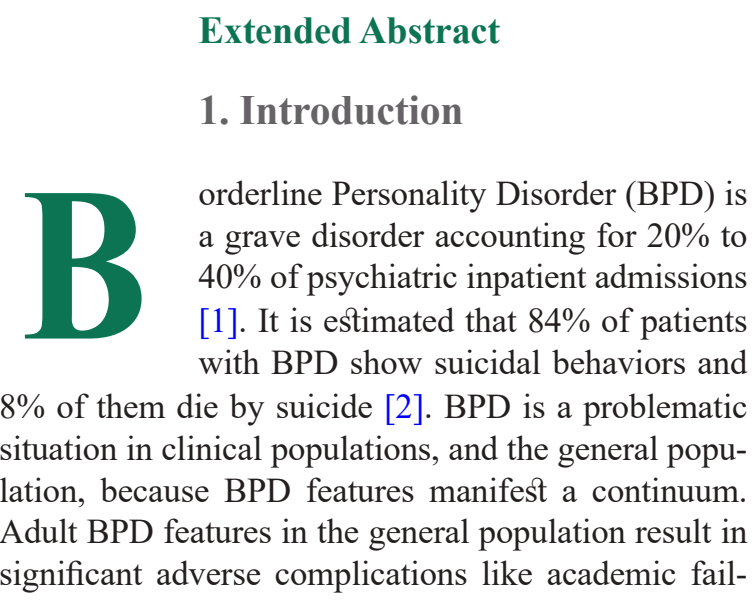

\section{A B S TRACT}

Objectives This study aimed to evaluate the mediating role of attachment styles and emotional regulation in the relationship of childhood emotional abuse and borderline personality disorder features.

Methods In a descriptive-correlational study, a sample of 312 students of Shahid Beheshti University was selected using cluster sampling method. Participants responded to the Child Trauma Questionnaire (CTQ), Borderline Personality Inventory (BPI), Difficulties in Emotion Regulation Scale (DERS), and Revised Adult Attachment Scale (RAAS). Data were analyzed using Structural Equation Modeling (SEM) of Path Analysis type.

Results Evaluation of the hypothesized model showed that this model has suitable fitness indices. Results showed that anxious attachment style and difficulties in emotion regulation can predict borderline personality disorder feature in full mediation.

Conclusion The result, supporting theoretical model for borderline personality disorder, proposes a suitable framework for etiology of the disorder.

ure, meeting lifetime criteria for a mood disorder, relationship problems, and alcohol use [3]

Several studies have confirmed the relationship between childhood emotional abuse and BPD in adulthood [4]. For example, Bierer et al. [40] found that childhood emotional abuse-and not physical or sexual abuse, or any form of neglect was predicted by a BPD diagnosis. However, although many studies have confirmed the relationship between childhood emotional abuse and BPD, this does not mean that all victims of emotional abuse are affected by this disorder [6]. Thus, it is important to delineate the specific mechanisms accounting for this relationship. In this regard, a hand-

* Corresponding Author:

Hossein Alizade, MSc.

Address: Department of Clinical \& Health Psychology, Faculty of Education and Psychology, Shahid Beheshti University, Teharan, Iran.

Tel: +98 (913) 3632185

E-mail: alizade72@yahoo.com 
ful of studies have identified two major links between childhood abuse and BPD pathology. One link is attachment style and insecurity in attachment [7] and the other is difficulties with emotion regulation [8].

Several empirical researches have shown the theoretical relationship between attachment insecurity and BPD [7]. However, there is no research agreement about which attachment styles are involved in this relationship [7]. The other link between child abuse and BPD is difficulties in emotion regulation. Studies suggest that children with a history of childhood emotional abuse are more likely to have difficulties with emotion regulation [3]. Similarly, individuals with BPD diagnosis report having general difficulties with emotion regulation [41].

Thus, this study aims to investigate the relationship between childhood emotional abuse and BPD features. In this regard, researchers intend to examine the role of attachment styles and emotion regulation. In other words, the role of attachment styles and emotion regulation in the relationship between childhood emotional abuse and BPD features has been studied.

\section{Method}

\section{Study participants}

A total of 312 students were selected from the Shahid Beheshti University, Tehran. The sample included $128(41.02 \%)$ male and $184(58.98 \%)$ female, with age ranging from 18 to 31 years $(\mathrm{M}=22.9, \mathrm{SD}=3.1)$. All participants gave their informed consent for this study.
Study measures

\section{Child Trauma Questionnaire (CTQ)}

CTQ is a 45-item retrospective self-report questionnaire that assesses subjectively participant's general childhood environment [10]. It covers five domains of childhood trauma, i.e., sexual abuse, emotional abuse, emotional neglect, physical abuse, and physical neglect. This study only employed the emotional abuse and emotional neglect subscales. The Persian version of CTQ showed a high internal consistency (Cronbach's $\alpha=0.65-0.91$ ) and acceptable test-retest reliability [11].

\section{Borderline Personality Inventory (BDI)}

BDI is a 53-item self-report inventory, which is widely used for assessing borderline personality traits in clinical and nonclinical samples. Persian version of BDI showed acceptable test-retest reliability $(\mathrm{r}=0.80)$ and acceptable subscales reliability [12].

\section{Difficulties in Emotion Regulation Scale (DERS)}

DERS is a self-report questionnaire that assesses both individual's overall difficulties in emotion regulation and their difficulties across six domains of emotion regulation. Persian version of this scale has a high internal consistency (Cronbach's $\alpha=0.86$ ) [13].

\section{Revised Adult Attachment Scale (RAAS)}

RAAS is an 18-item self-report questionnaire which assesses three attachment styles: secure attachment, insecure anxious attachment, and insecure avoidant attachment. Cronbach's $\alpha$ of the Persian version for each of the three subscales

Table 1. Matrix of correlation between all variables

\begin{tabular}{|ccccccc}
\hline Variables & $\mathbf{1}$ & $\mathbf{2}$ & $\mathbf{3}$ & $\mathbf{4}$ & $\mathbf{5}$ & $\mathbf{6}$ \\
\hline 1. Childhood emotional abuse & 1 & & & & & \\
2. Secure attachment style & 0.005 & 1 & & & & \\
\hline 3. Insecure avoidant attachment & $0.33^{* *}$ & 0.047 & 1 & & 1 & 1 \\
\hline 4. Insecure anxious attachment & $0.41^{* *}$ & 0.038 & $0.023^{* *}$ & 1 & $0.031^{* *}$ & 1 \\
\hline 5. Difficulties in emotion regulation & $0.036^{* *}$ & 0.022 & $0.011^{*}$ & $0.38^{* *}$ & $0.43^{* *}$ & 1 \\
6. BPD features & 0.010 & 0.034 & 0.06 & Iranian Journal of \\
PSYCHATRY AND CUNICAL PSYCHOLOGY
\end{tabular}

Table 2. Measures of goodness of fit of structural equation modeling

\begin{tabular}{cccccccc}
\hline Index & $\mathbf{X}^{2} / \mathrm{df}$ & RMSEA & GFI & AGFI & NFI & CFI & RFI \\
\hline Value & 1.678 & 0.056 & 0.929 & 0.884 & 0.929 & 0.970 & 0.956 \\
\hline
\end{tabular}




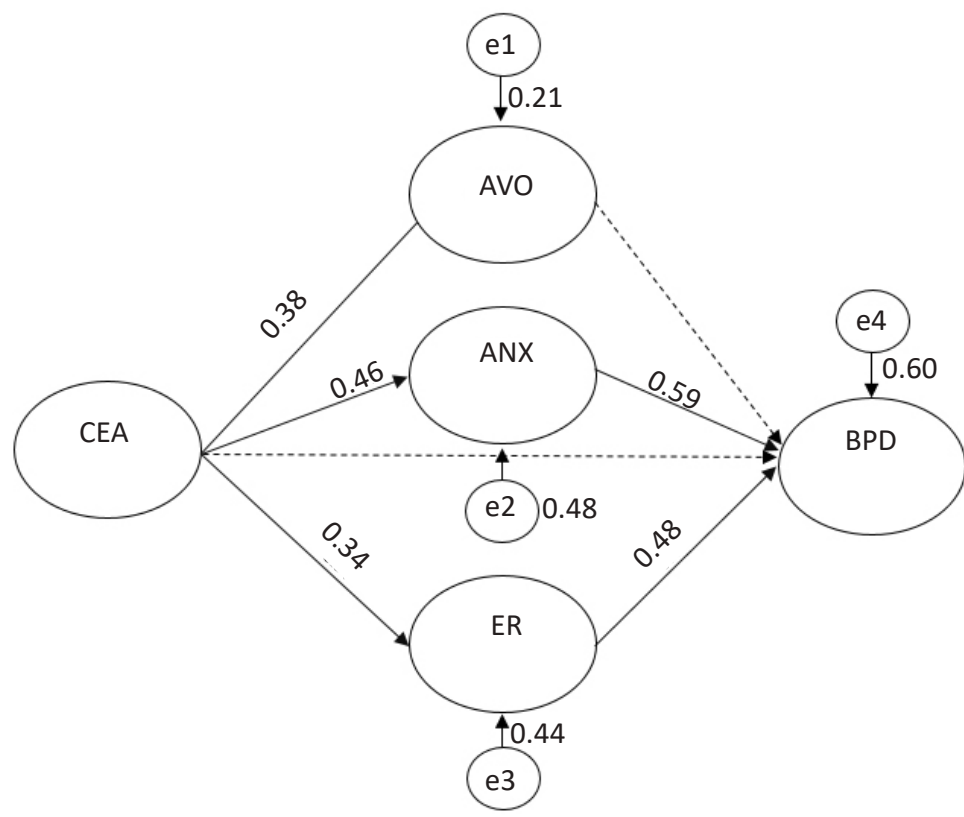

Figure 1. Measurement model of variables

Insignificant paths are in dashed lines; CEA: Childhood Emotional Abuse; AVO: Avoidant Attachment Style; ANX: Anxious Attachment Style; ER: Emotion Regulation; BPD: Borderline Personality Disorder Features

are $0.81,0.85$ and 0.81 , respectively, which are high. Also test-retest coefficient of this scale is acceptable $(\mathrm{r}=0.89)$ [14].

\section{Data analysis}

The present study is a correlational study and its data were processed by Structural Equation Modeling (SEM) or causal modeling using path analysis.

\section{Results}

Table 1 demonstrates matrix of correlations between variables of the study. According to Table 1, as the direct path of emotional abuse and BPD was not significantly correlated, hence this path was removed in the final model and the measurement model was again evaluated. Fit indices (Table 2) demonstrated a good fit suggesting that this model of measurement offers an acceptable representation of the model. The final model (Figure 1) demonstrates no direct path between childhood emotional abuse and BPD; however, this relationship is confirmed through a non-direct path. In other words, both attachment styles and also difficulties in emotion regulation have fully-mediated the relationship between childhood emotional abuse and BPD.

\section{Discussion}

The current study aims to investigate the SEM analysis of the relationship between childhood emotional abuse and BPD with mediating roles of attachment styles as well as difficulties in emotion regulation in Iranian student samples.
Findings of the current study show that attachment styles and difficulties in emotion regulation can fully mediate the relationship between childhood emotional abuse and BPD, which are consistent with the findings of Kuo et al. [3], Neumann [15] and Critchfield et al. [16]. Furthermore, our model was statistically confirmed and is also consistent with Kuo et al. [42]. The only exception is that Kuo model suggests that difficulties in emotion regulation can partially mediate the relationship between childhood emotional abuse and BPD, but our model suggests complete mediation between these variables. According to our model, insecure anxious attachment can solely predict BPD along with experiencing emotional abuse in childhood. Also, difficulties in emotion regulation can predict BPD.

The child after being continually exposed to emotional abuse goes through developmental trajectories and pathways which make the child prone to BPD [18]. Two developmentally important pathways are attachment-based relationships and the development of emotion regulations. Current research shows that participants who had difficulties in this domains were more likely to have BPD features.

\section{Ethical Considerations}

\section{Compliance with ethical guidelines}

First, the general explanations of the research objectives were given to the participants and after receiving informed consent from them, test questions were distributed among them. 


\section{Funding}

This research received no specific grant from any funding agency in the public, commercial, or not-for-profit sectors.

\section{Conflict of interest}

All authors declared no conflict of interest.

\section{Acknowledgements}

We would like to specially acknowledge Dr. Mohsen Moradi for statistical consultations. Also, we acknowledge the participants of this research. 


\title{
تجربه آزار هيجانى كودكى و ويزّكى هاى اختلال شخحصيت مرزى در بزرَّالى: نقش واسطهاى

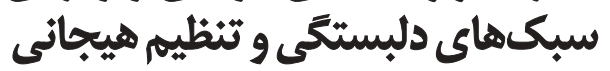

\author{
حسين يورشهريار'، "خسين علىزاده'، كاظم رجايىنيا"

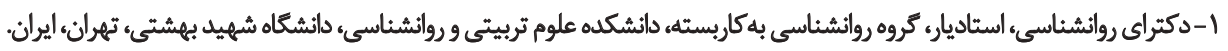

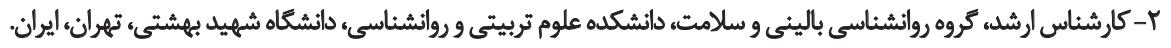

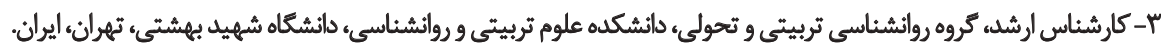

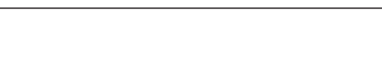

مورد بحث بين نظريهيردازان و محققان به شمار مى آيد [ץ]].

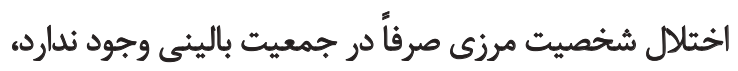

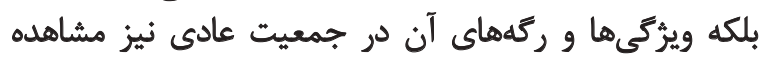

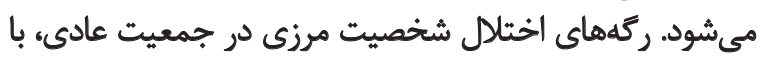

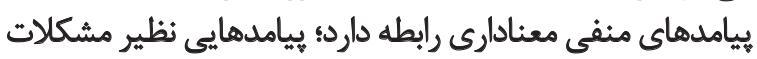

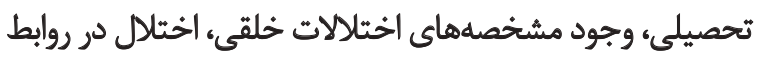

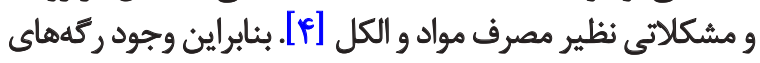

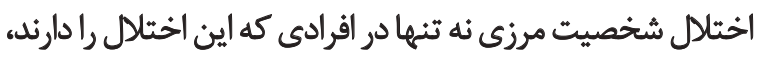

بلكه در جمعيت عادى نيز باعث ايجاد مشكلاتى مئش إشود.

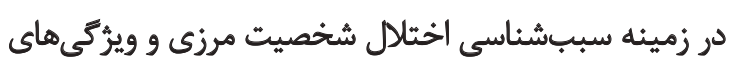

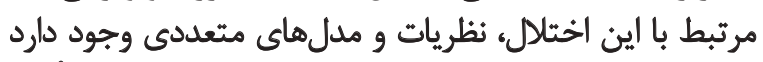

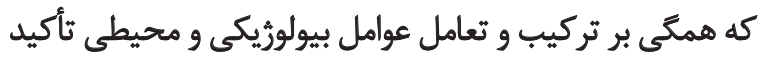

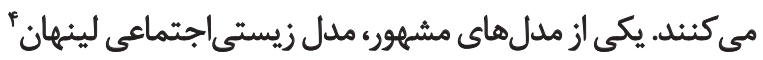
است [f]. او معتقد است اختلال شخصيت مرزى، در نتيجه

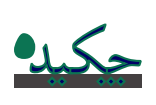

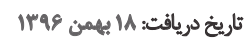

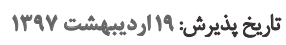

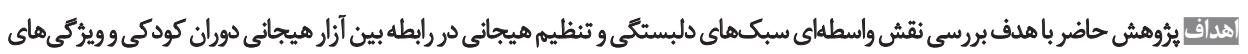
اختلال شخصيت مرزى انجام شده است.

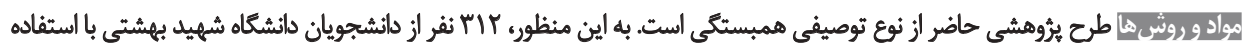

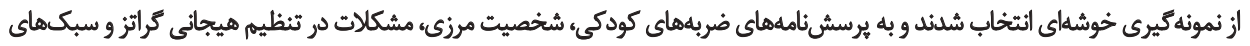

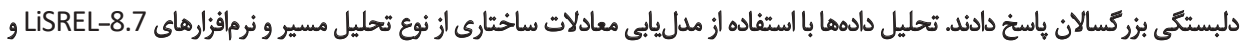
AMOS-23 أنجام شد.

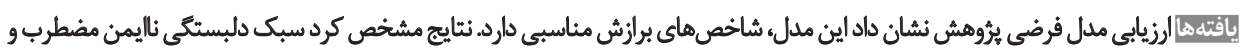

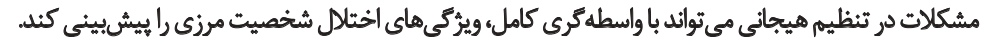

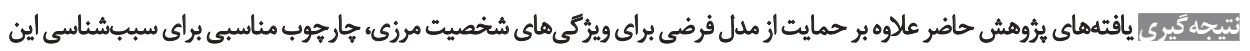
اختبلال ارائه مي دهد.

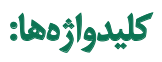

سبكهاي دلبسيتكي، اختلال شخصيت مرزى، البسئي، تنظيم هيجاني، آزار هيجائي، مدلياني هيجي معادلات ساختارى

مقدمه

اختلال شخصيت مرزى'، اختلال شديد و ناتوان كنيدهاى است

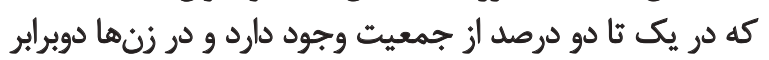

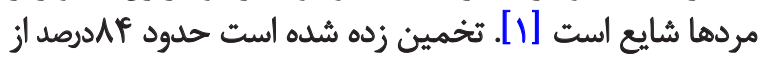

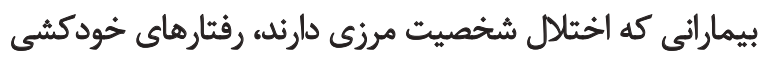

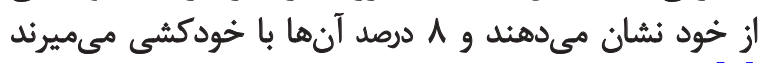

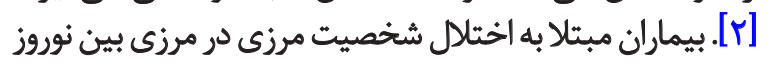

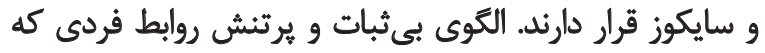

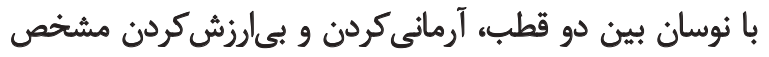

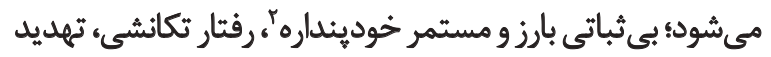

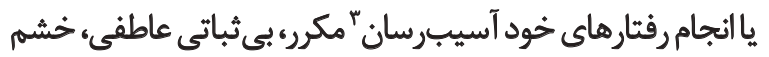

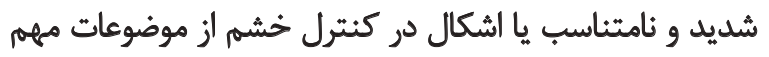

1. Borderline personality disorder

2. Self-concept

3. Self-injury

\section{-}

*ويند : توينده مسئول:

حسين علىزادها

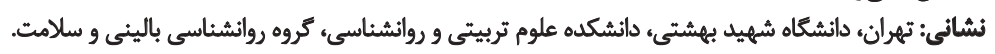
تلفن: يست الكترونيكي: alizade72@yahoo.com 


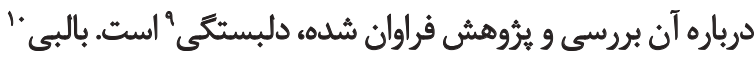

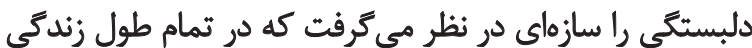

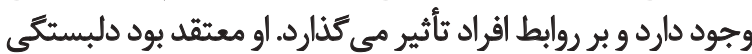

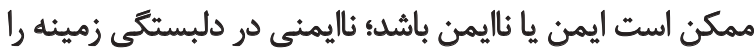

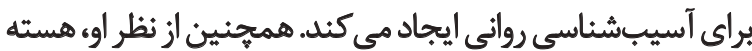
اختلالات شخصيت، دلبستخى نايمن است [11]

يثروهشهاى تجربى متعددى، رابطه نظرى بين نايمنى در

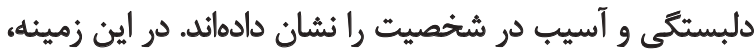

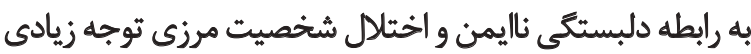

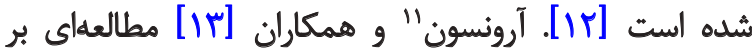
آزمودنىهاى بالينى انجام دادند كه نتايج نشان داد باد بين اختان آنال

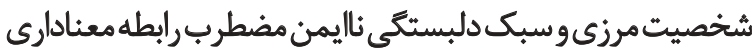

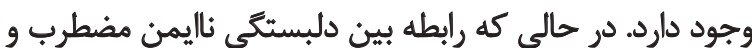

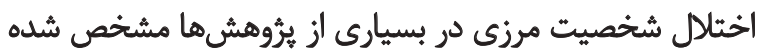
است، درباره رابطه بين سبك دلبستئى ناليمن اجتنابي و واختيلال

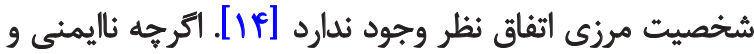

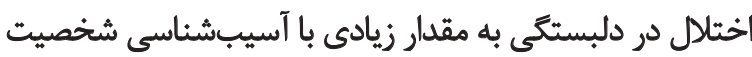

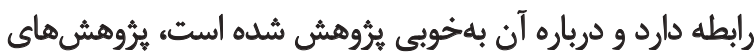

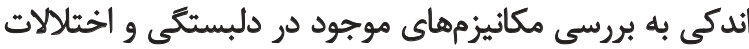

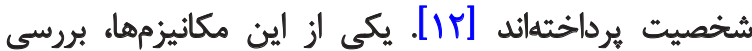
راهبردهاى تنظيم هيجانى در افراد است.

تنظيم هيجان به فرايندى اطلاق مي شيود كه فرد بداند تحت

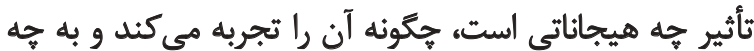

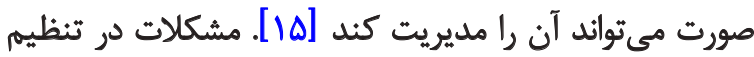

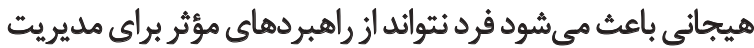

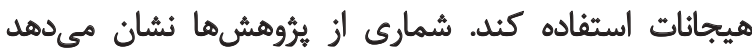

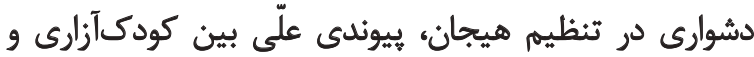

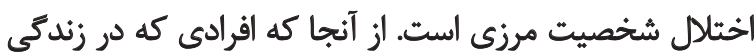

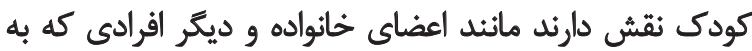

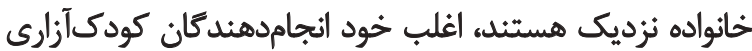

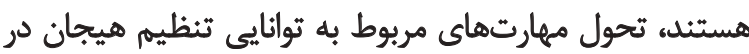

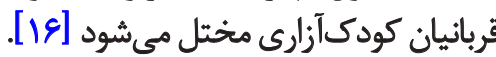

علاوه بر آن، يافتههاى برُوهشى اخير نشان مى ندهد آزار

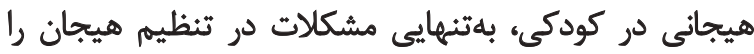

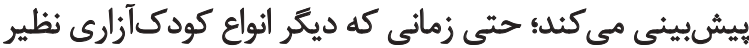

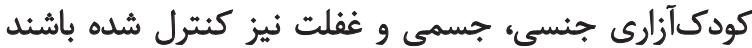

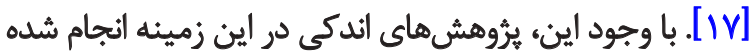

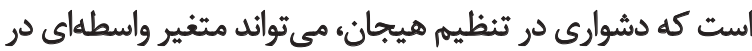

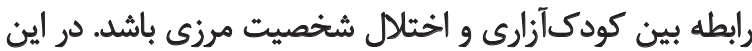

9. Attachment

10. Bowlby

11. Aaronson
تعامل و تبادل بين آمادكى زيستى فرده مشكلاتى در زمينه تنظيم هيجانى و محيط تربيتى آشفته به وجود مي آيد.

مدل هاى ديكر نيز به طور مشابه به نقش تعيين كننده محيط

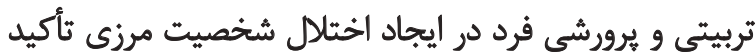

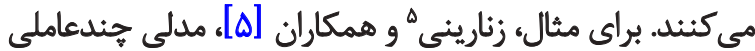

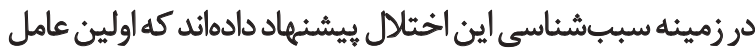

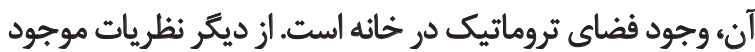

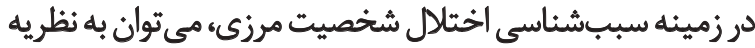

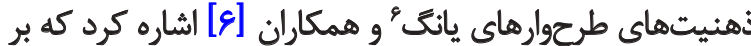

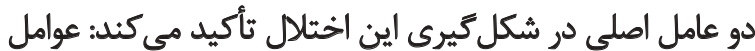

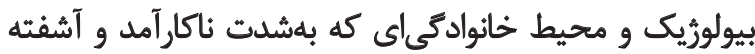

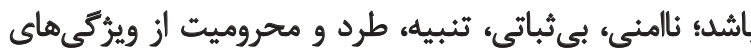

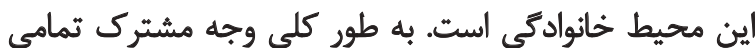

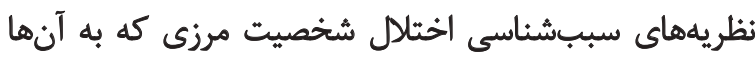

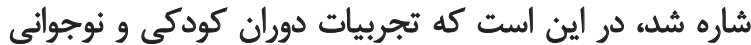

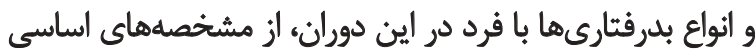

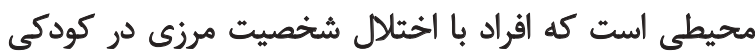

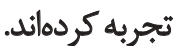

همخوان با اين نظريات، يُروهشهاى زيادي صورت كرفته است

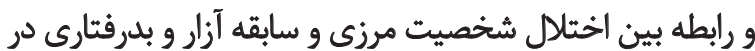

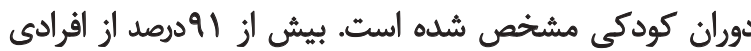

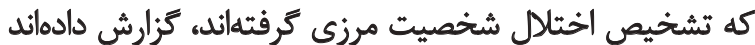

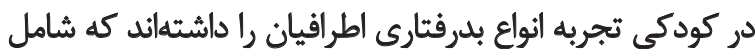

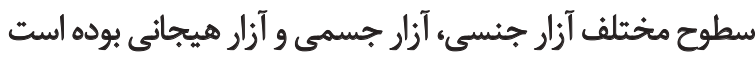

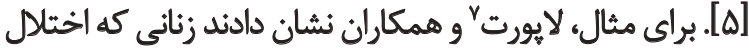

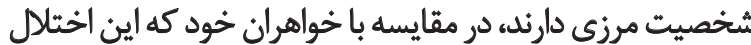

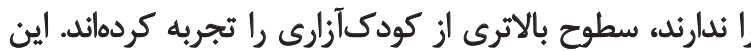

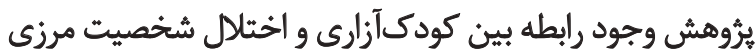

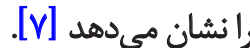

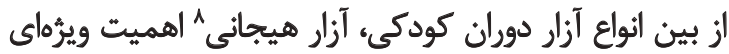

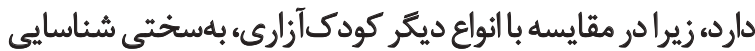

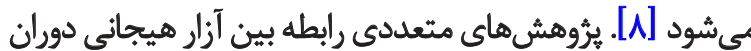

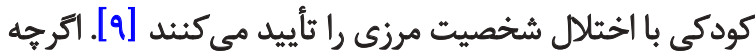

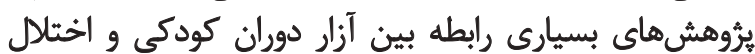

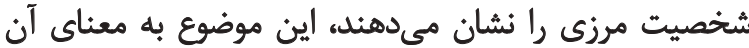

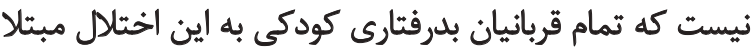

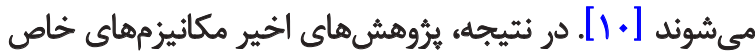

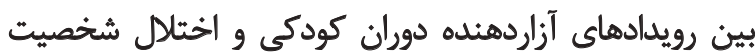

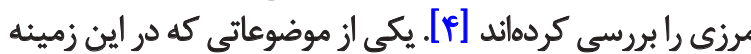

5. Zanarini

6. Young

7. Laporte

8. Emotional abuse 
كرفتن رضايت كتبى از ايشانء سؤالات آزمون بين آنها توزيع آنواني

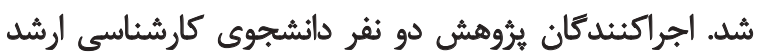

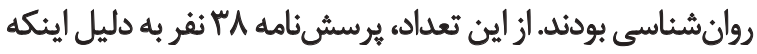

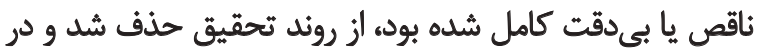

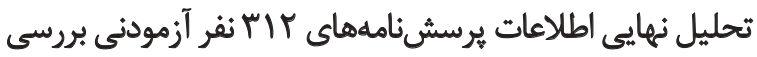

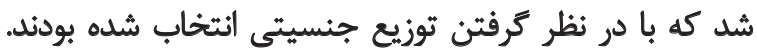

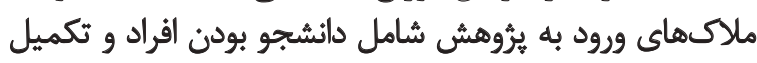

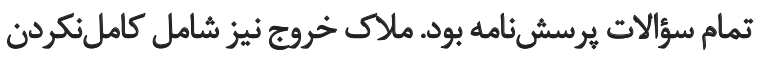
يك سؤال و بيشتر و ياسخ تويى تصادفى بودي.

ابيزار ابزارهاى تردآورى اطلاعات به صورت زير است:

\section{يرسش نامه ضربه هاي كودكى "}

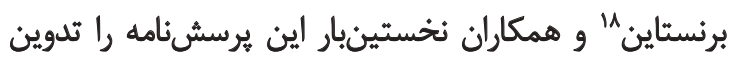

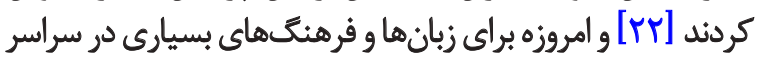

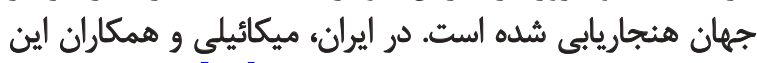

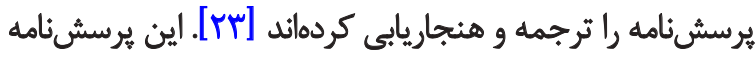

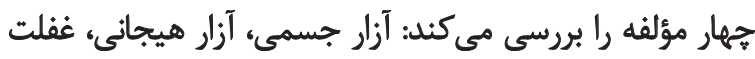

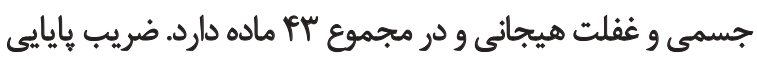

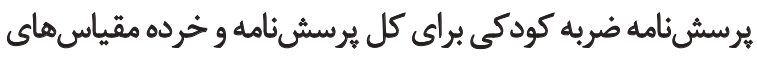

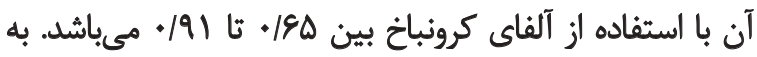

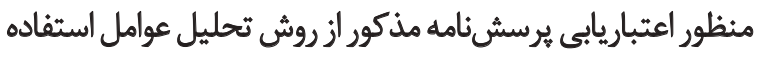

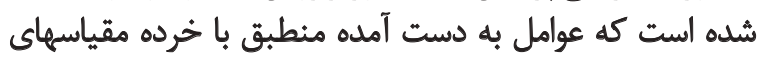

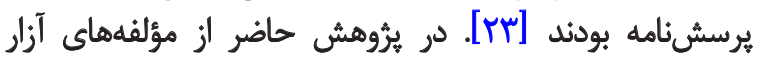

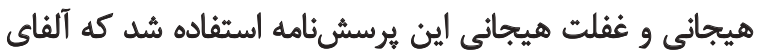

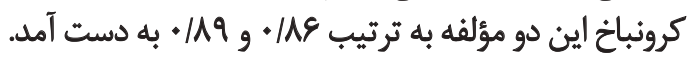

\section{يرسش نامه شخصيت مرزى"}

اين يرسشنامه براى سنجش صفات شخصيت مرزى در

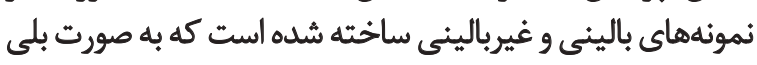

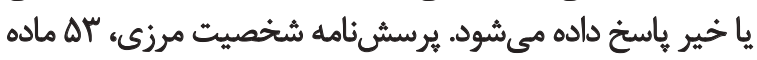

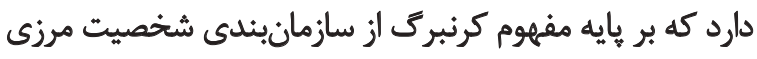

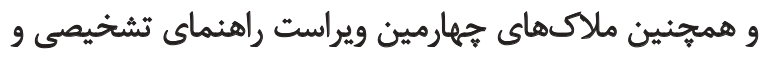

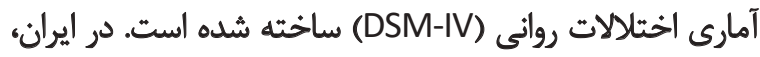

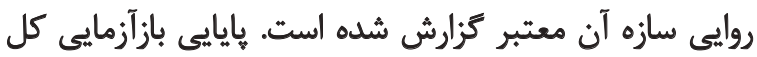

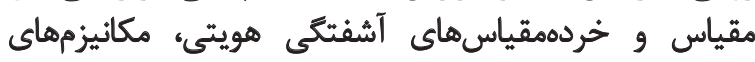

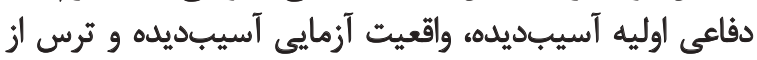

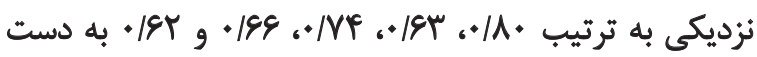

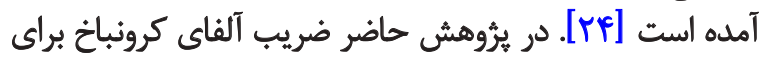

17. Child Trauma Questionnaire (CTQ)

18. Bernstein

19. Borderline Personality Inventory (BPI)

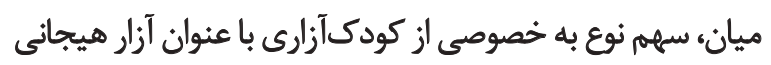

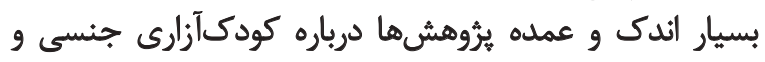
جسمى انجام شده است.

ورئوهش حاضر قصد دارد رابطه بين آزار هيجانى دوران كودكي

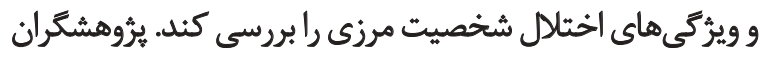

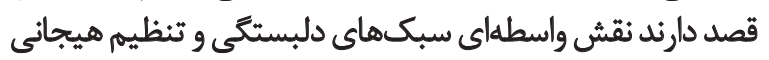

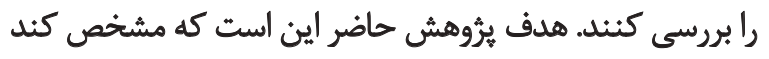

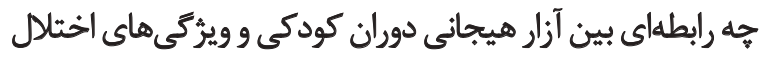

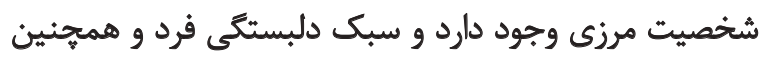

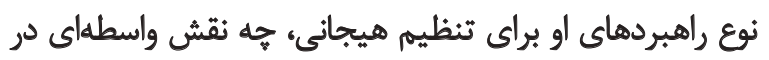

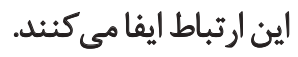

موش

هروهش حاضر از نوع توصيفى است و از روش مدليابي

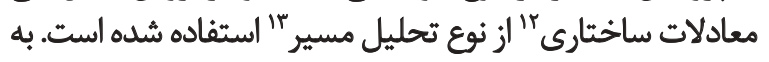

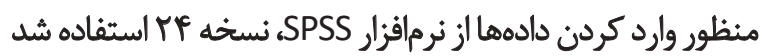

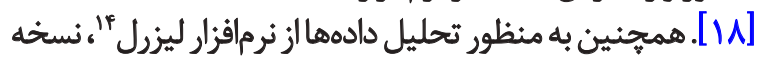

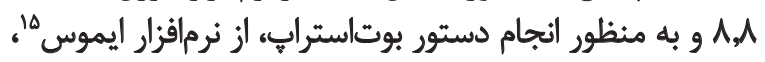

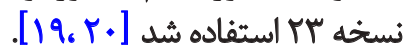

$$
\text { آزمودنى ها }
$$

جامعهى آمارى يُورهش حاضر، شامل دانشجويان دختر و يسر كاري

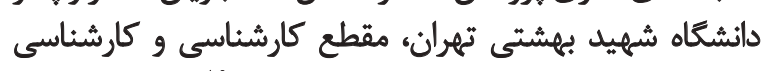

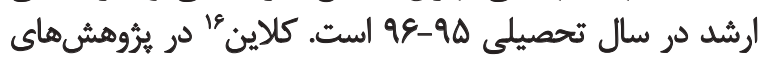

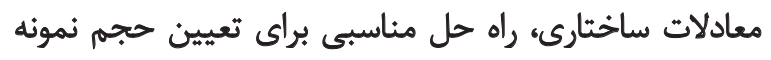

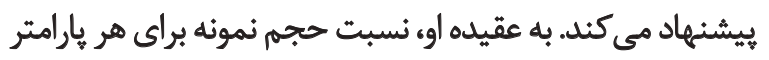

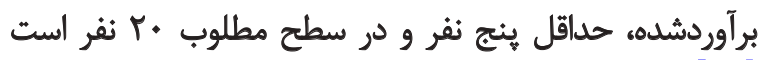

[ [

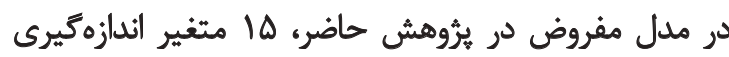

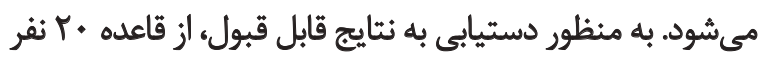

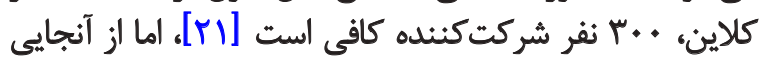

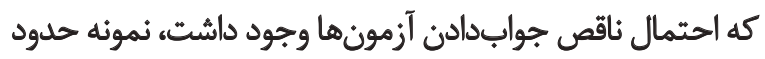

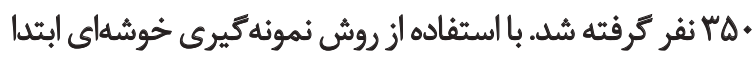

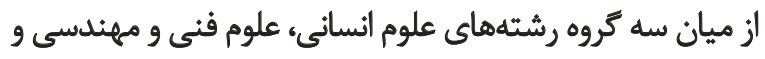

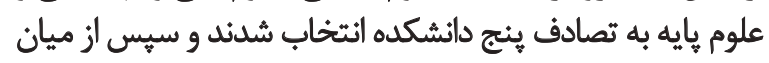

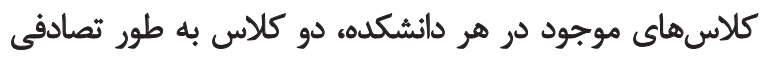

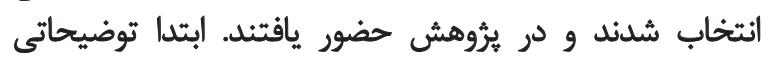

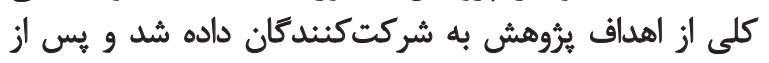

12. Structural equation modeling

13. Path analysis

14. Lisrel

15. Amos

16. Kline 


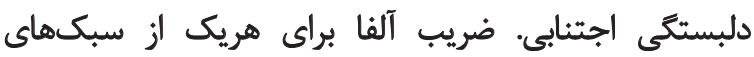

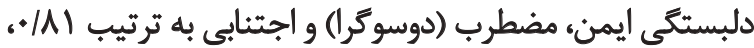

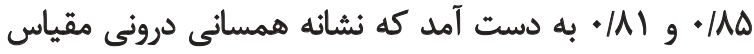

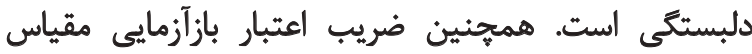

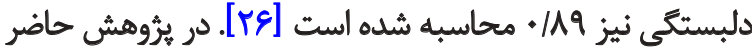

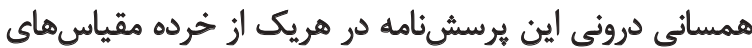

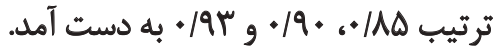

يافتهها

مشخصات جمعيتشناختى نمونه تحقيق در جدول شماره

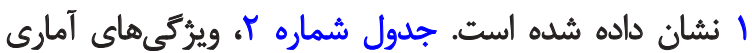

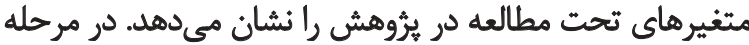

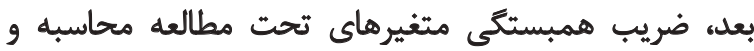

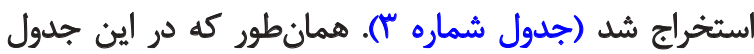

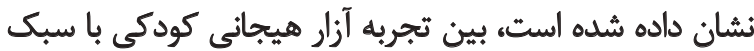

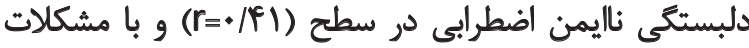

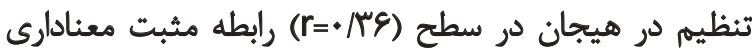

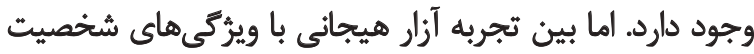

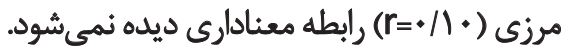

در مرحله بعد مدل مدنظر ارزيابى شد. بيش از ارزيابى

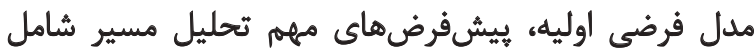

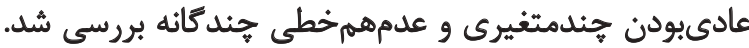

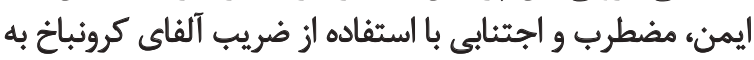

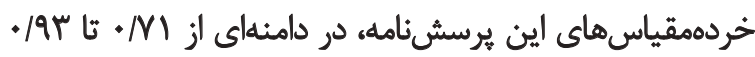
به دست آمد.

\section{"يرسش بامه مشكلات تنظيم هيجانى كراتز”}

مقياس دشوارى در تنظيم هيجانى، مقياسى عب مادمايى

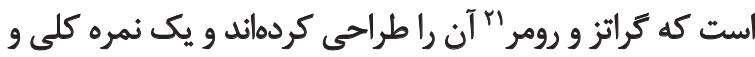

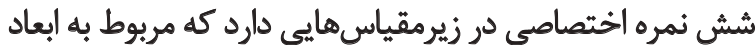

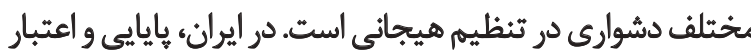

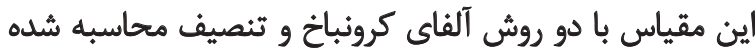

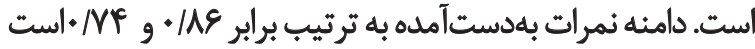

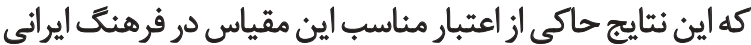

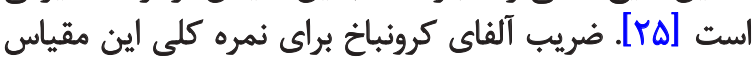

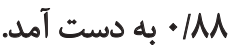

يرسش نامهسبكهاى دلبستكىبزرتسالان تجديدنظرشده" كولينز و ريد" اين يرسشنامه را بر اساس نظريه هازان و ورئ

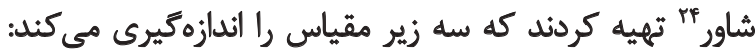
سبك اضطرابى (دوسوكرا)، سبك نزديكبودن زير (ايمن) و سبك ميك

20. Difficulties in Emotion Regulation Scale (DERS)

21. Gratz \& Roemer

22. Revised Adult Attachment Scale (RAAS)

23. Collins \& Read

24. Hazen \& Shaver

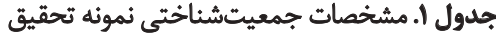

\begin{tabular}{|c|c|c|}
\hline ميانكين سنى (انحراف استاندارد) & تعداد (درصد) & \\
\hline$r r / \Delta E(r / P T)$ & $\operatorname{IVe}(\Delta \Delta / N)$ & زن \\
\hline$M F / * N(r / \varphi \Delta)$ & $\mid \Psi A(\varphi \varphi / T)$ & مرد \\
\hline$T r / M q(T / \Delta T)$ & rir(1). & كل \\
\hline
\end{tabular}

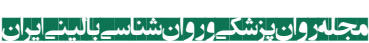

جدول ז. شاخص هاي مركزى و هراكندكى متغيرهاي مورد مطالعه

\begin{tabular}{|c|c|c|c|c|}
\hline ييشترين & كمترين & آزمون كالموكرف - اسميرونف & ميانكين (انحراف استاندارد) & مثغيرها \\
\hline ra & 1 & $1 / \circ 0$ & $\left.1 \Delta / R^{+}\right)(\Delta / \pi)$ & ويرَكى هاى شخصيت هرزى \\
\hline TA & $r$ & $1 / \mathbb{T H}^{2}$ & $Q / T A(1 / \cdot \Delta)$ & تجربه آزار هيجانى كودكى \\
\hline 1 ipq & il & $1 / m e$ & $N \& / Y(Q / V A)$ & دشوارى در تنظيم هيجائي \\
\hline re & f & $1 / \cdot r$ & $K M / \Delta q(P / I E)$ & سبك دلبستى ايمن \\
\hline$M$ & $r$ & $1 / \Delta f^{f}$ & $q / N \Delta(T / A V)$ & سبك دلبستكى ثاليمن اضطر ابيى \\
\hline M & f & $\mid / M$ & IY/EY $(T / I Y)$ & سبك دلبستكى ناليمن اجتنايي \\
\hline
\end{tabular}


جدول ؟. ضريب همبستئى بيرسون متغيرهاي مورد مطالعه

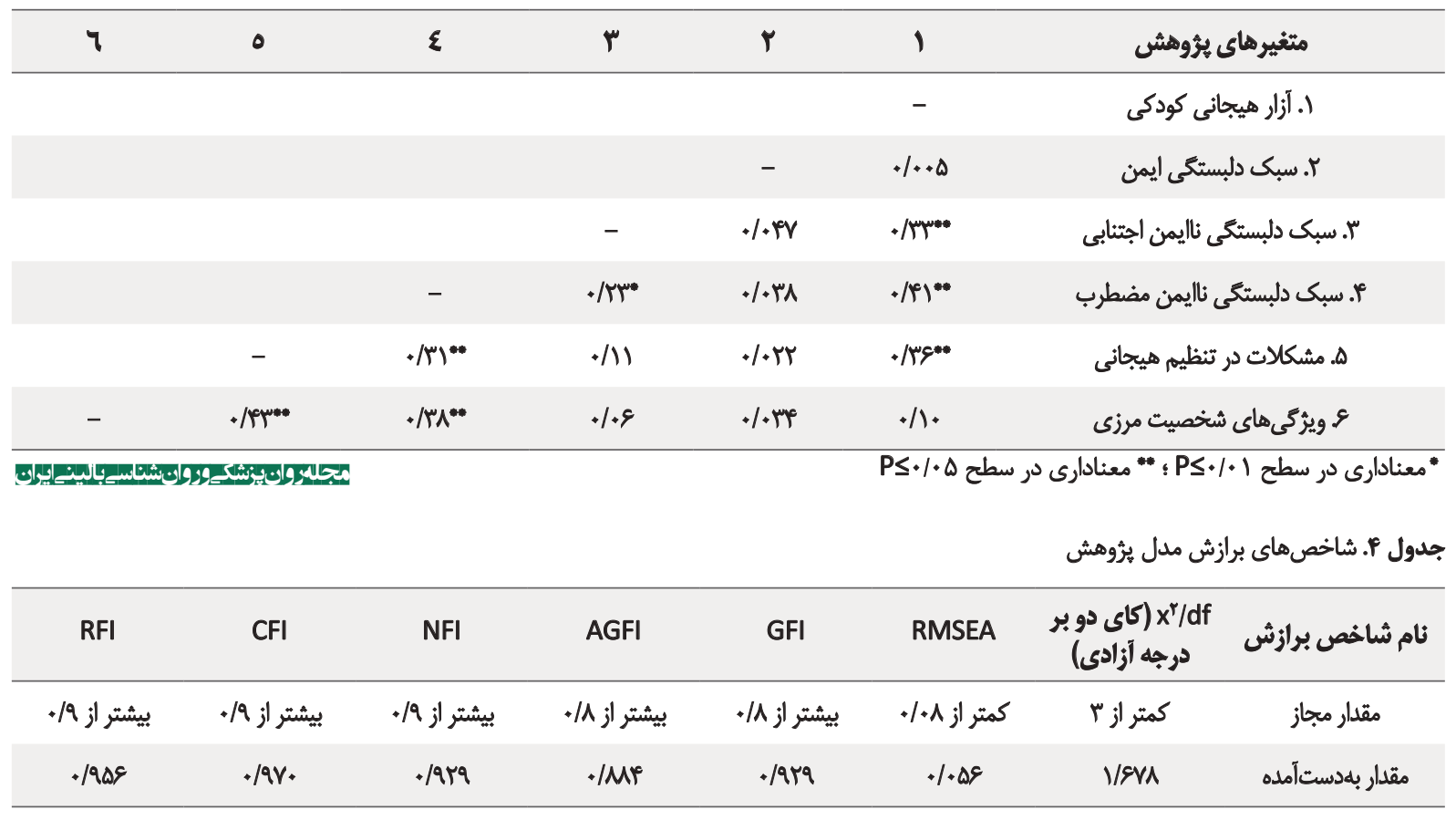

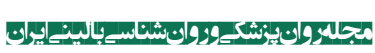

كه در جدول شماره F نشان داده شده است، شاخصهاى برازش نشاندهنده برازش مناسب اين مدل است.

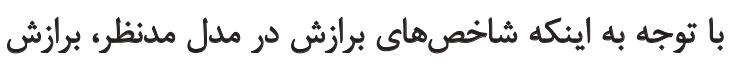

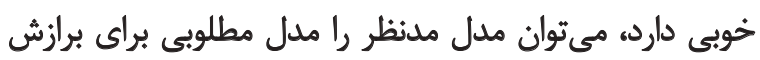

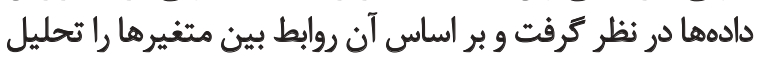

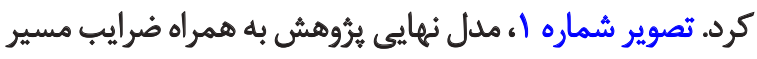
آن رانشان مي نهودير شماره.

به منظور بررسى مسيرهاى غيرمستقيم مدل، از دستور

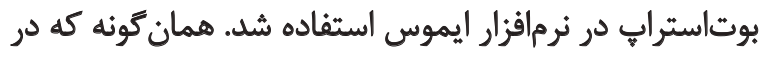

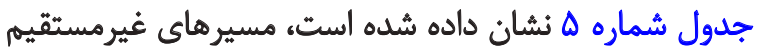

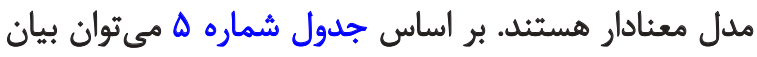

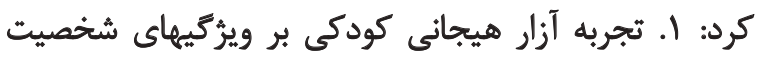

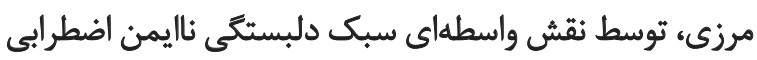

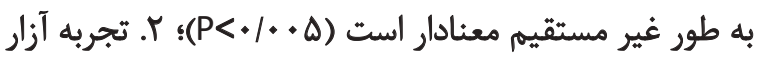

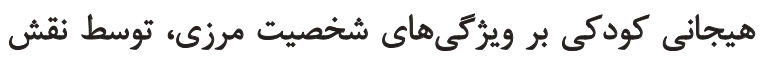

فرضيه عادىبودن خندمتغيرى با محاسبه شاخص كشيدكى

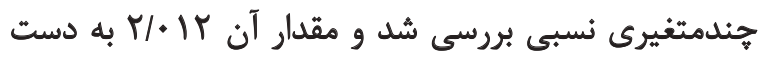

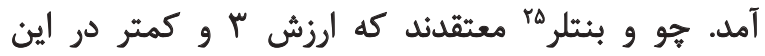

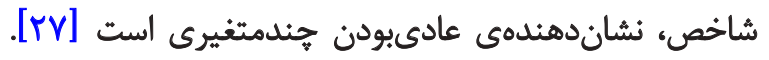

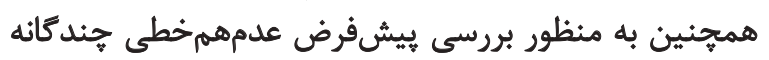

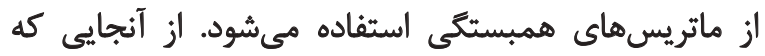

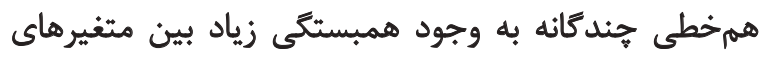

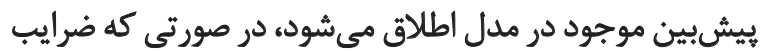

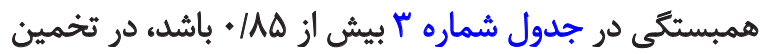

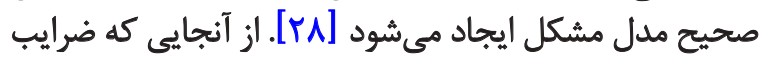

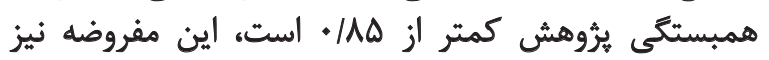

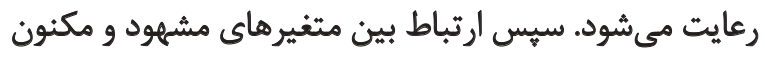

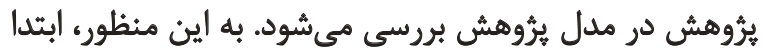

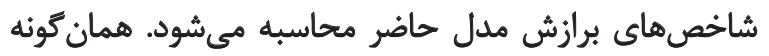

25. Chou, \& Bentler

جدول ه. مسير هاى بوتاستراب براي مسيرهاى مدل

\begin{tabular}{|c|c|c|c|c|}
\hline \multirow{2}{*}{ سطع معنادارى } & \multirow{2}{*}{ مقدار اثر } & \multicolumn{2}{|c|}{ بوتاستراب } & \multirow{2}{*}{ 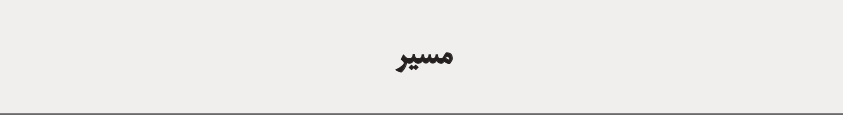 } \\
\hline & & هد بالا & هد بايين & \\
\hline$+1+\infty$ & $\cdot / \pi \Delta$ &.$/ 4 T \Delta$ &.$/ M I V$ & تجربه آزار هيجانى كودكى ـ دلبستكى ناليمن اضطرابى ـ ـويزكى هاى شخصيت مرزى \\
\hline$\%+\Delta$ & $\bullet / r^{\prime \prime}$ & $\cdot / \pi v \Delta$ & $+/ 1 r q$ & تجربه أزار هيجانى ـ ـشوارى در تنظيمه هيجانى ـ ويزّكى هاى شخصيت مرزى \\
\hline
\end{tabular}




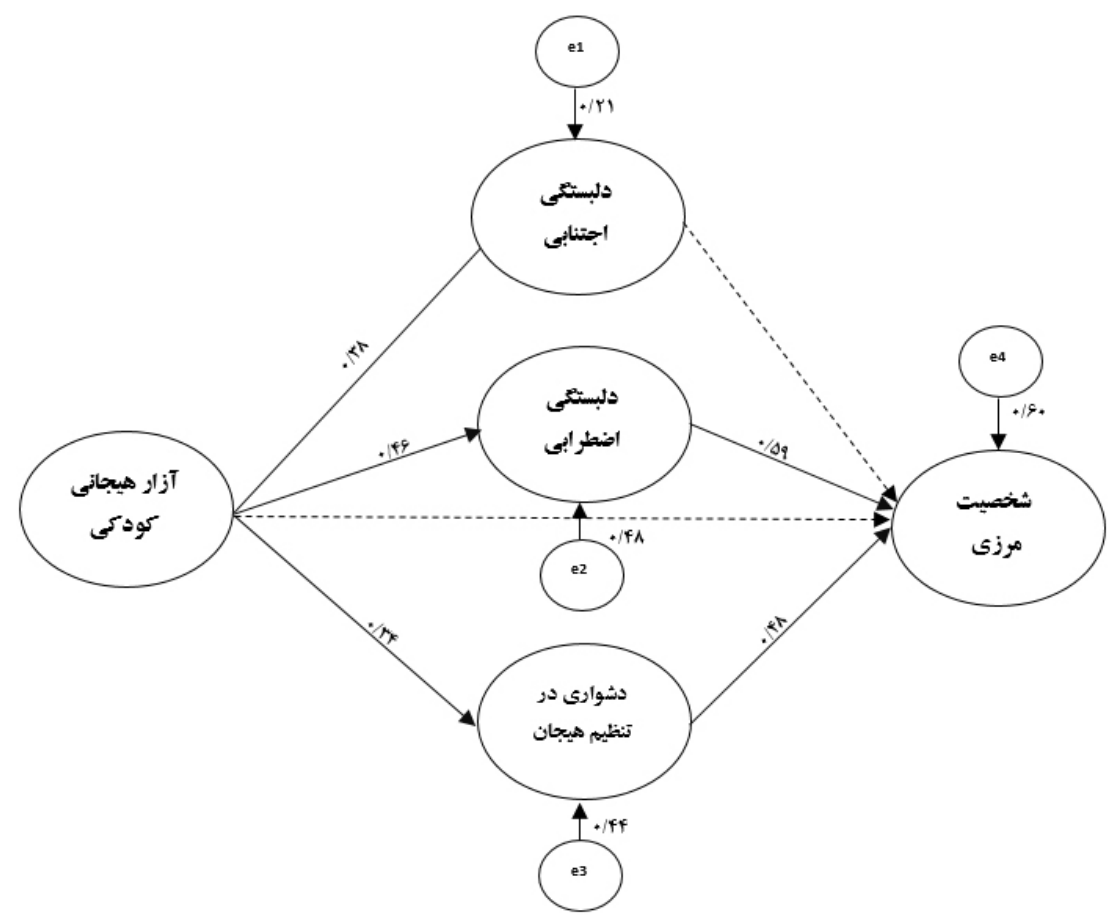

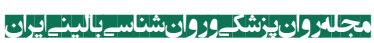

تصوير ا. مدل نهايي در رابطه آزار هيجائى كودكى و شخصيت مرزى

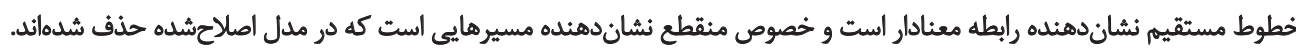

همجئين حذف شدن مسير مستقيهم آزار هيجانى در بيشبيشينى

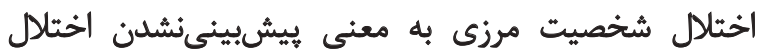

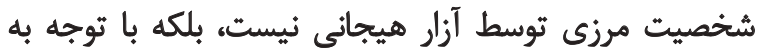

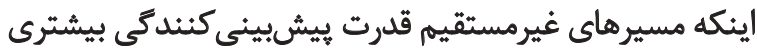

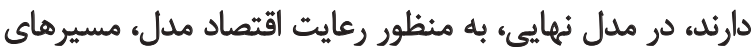

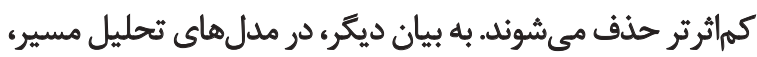

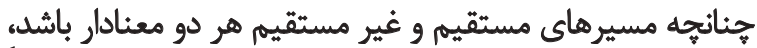

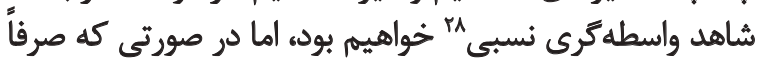

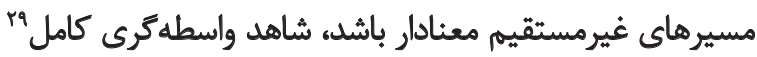

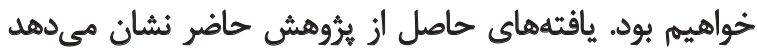

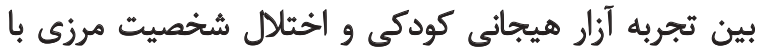

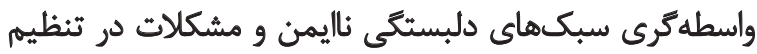

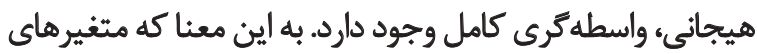

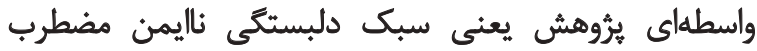

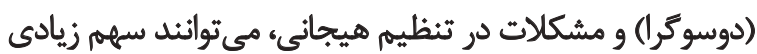

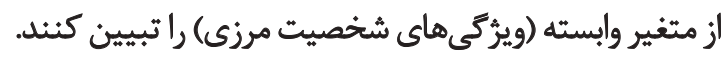

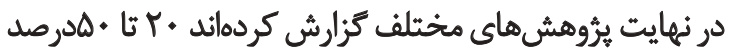

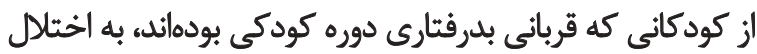

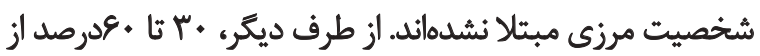
افرادى كه مبتالا به اختّلال شخصيت مرزى هستيند، هيج نوع
واسطهاى دشوارى در تنظيم هيجان به طور غيرمستقيم

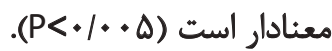

\section{+os!}

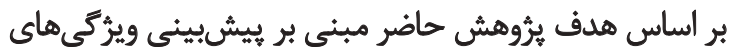

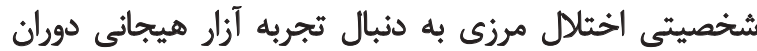

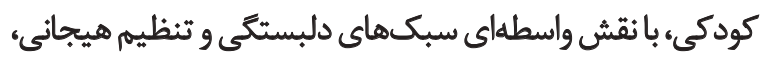

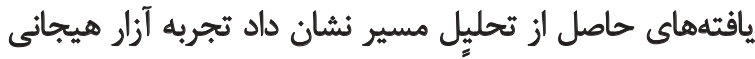

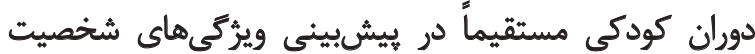

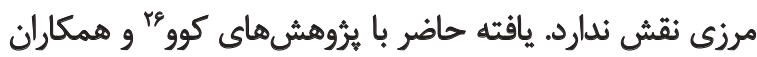

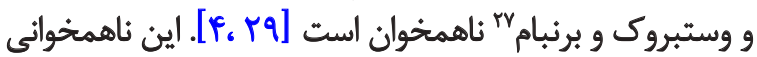

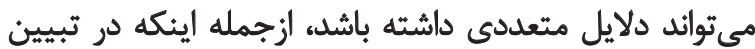

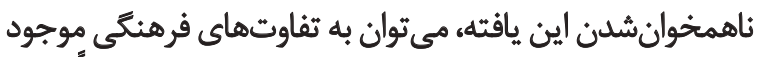

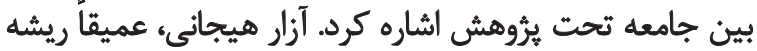

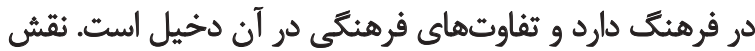

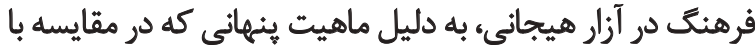

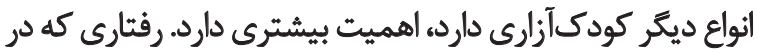

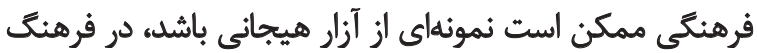

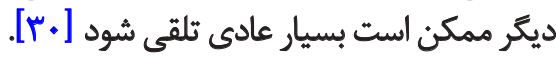

26. Kuo

27. Westbrook \& Berenbaum 


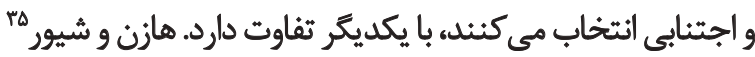

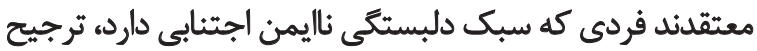

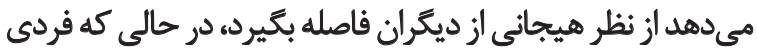

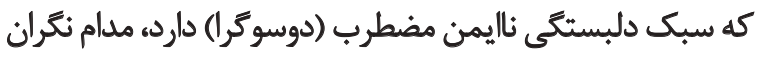

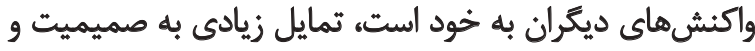

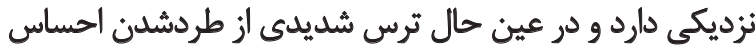

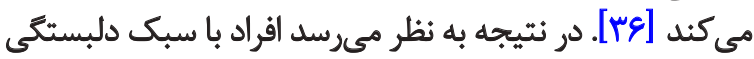

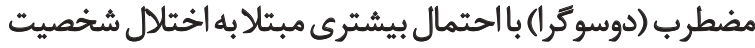

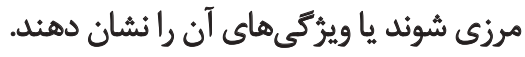

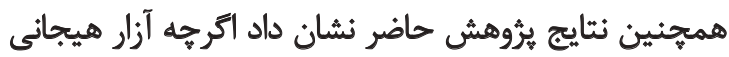

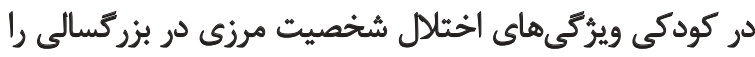

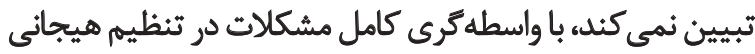

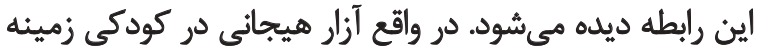

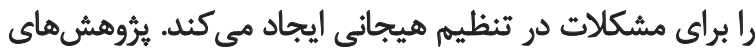

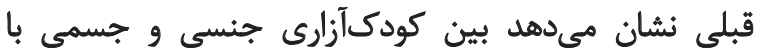

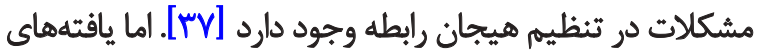

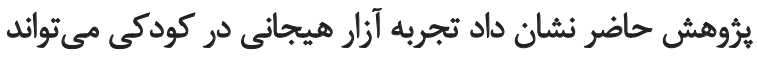

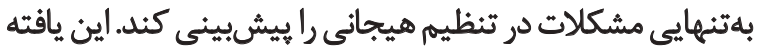

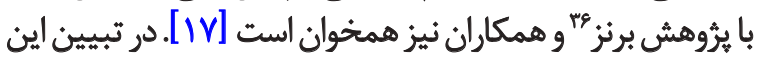

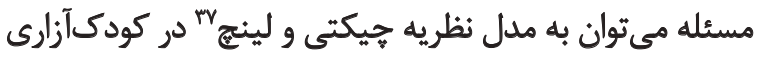

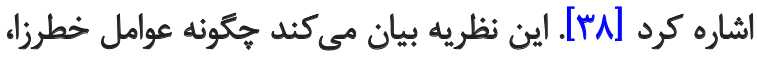

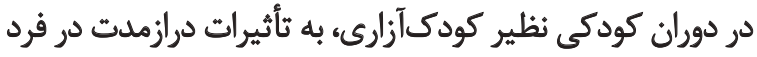

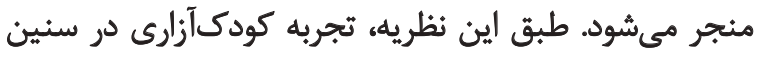

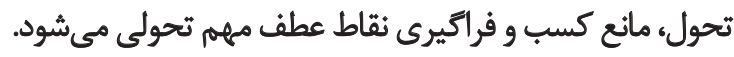

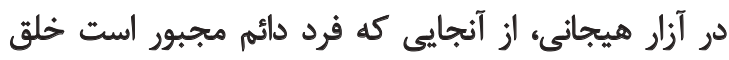

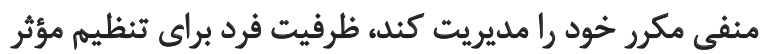

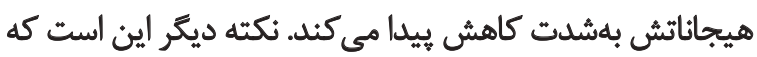

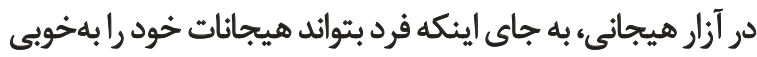

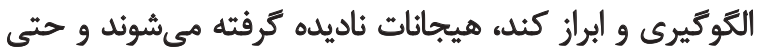

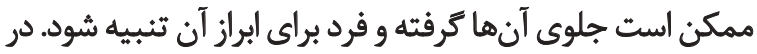

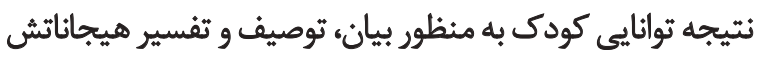

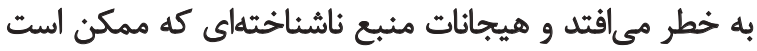

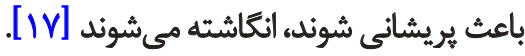

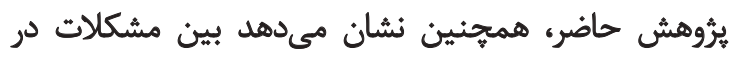

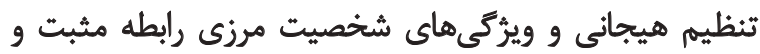

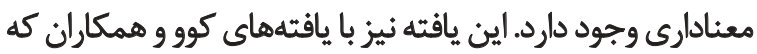
نقش واسطه

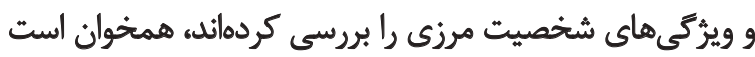

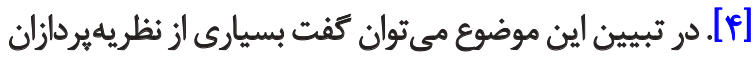

35. Hazan \& Shaver

36. Burns

37. Cicchetti \& Lynch

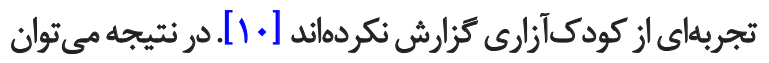

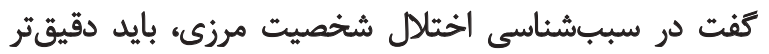

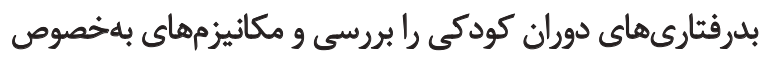

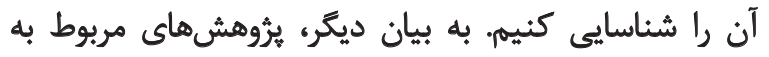

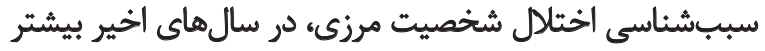

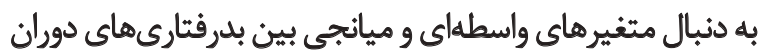

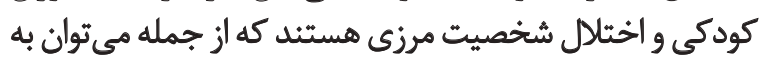
سبكهاى دلبستكى و مشكلات تنظيمه هيجانى اشاره كرد. يثروش حاضر نشان مى دهد آزار هيجانى دوران كودكى به

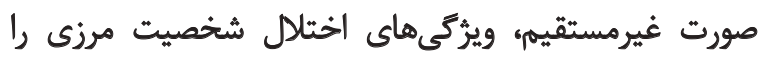

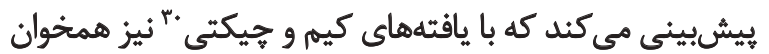

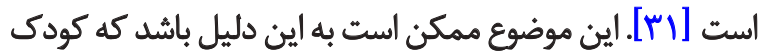

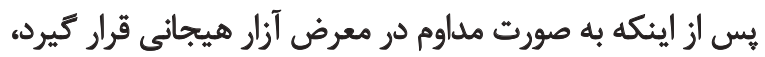

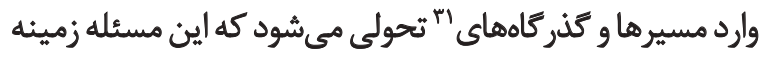

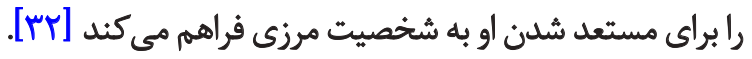
يكى از اين كذر كاههاى تحولى، سبك دلبستى نائ نايمن است.

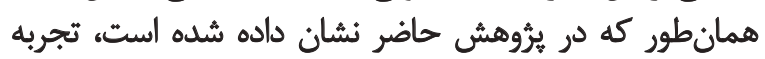

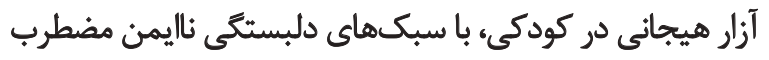

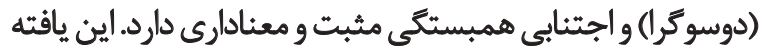

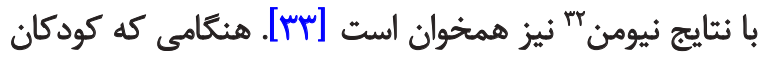

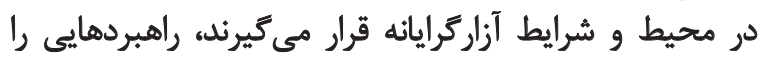

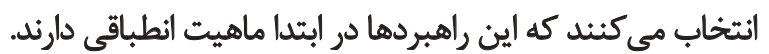

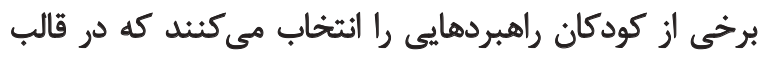

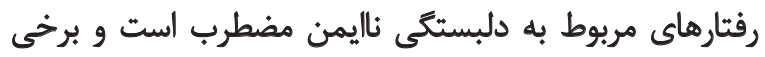

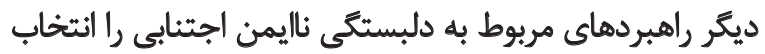

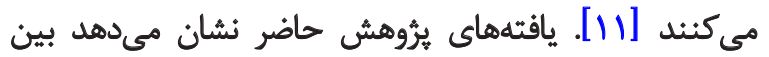

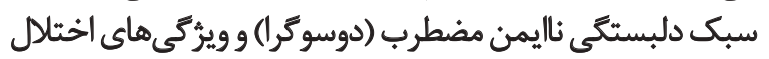

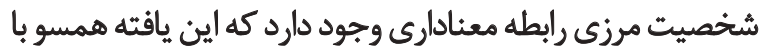

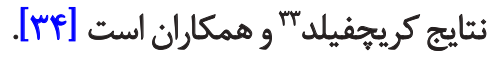

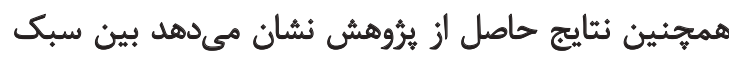

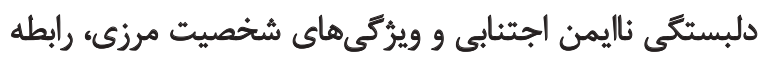

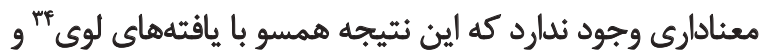

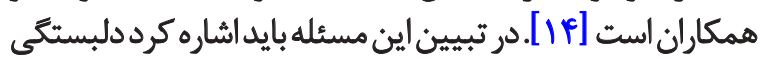

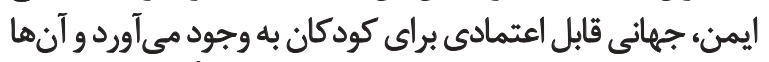

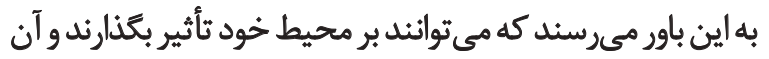

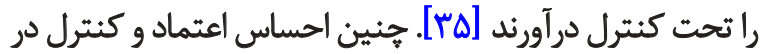

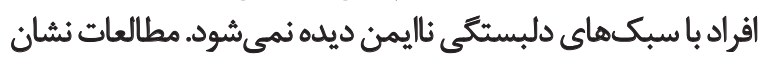

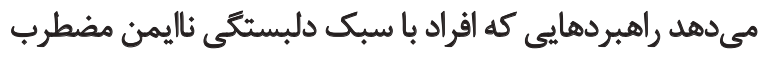

30. Kim \& Cicchetti

31. Trajectory

32. Neumann

33. Critchfield

34. Levy 


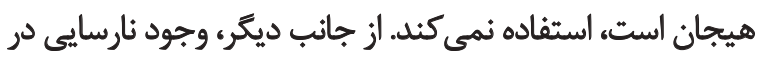

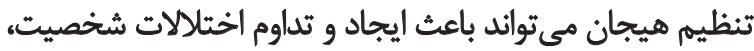

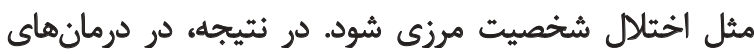

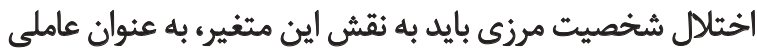

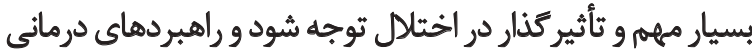
در اين خصوص به كار ترفته شود.

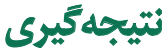

به طور كلى، نتايج يُروهش حاضر نشان داد وجود سبكهاي

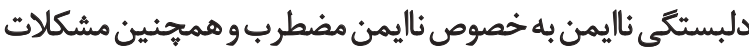

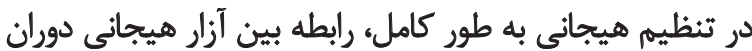

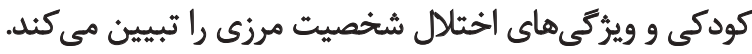

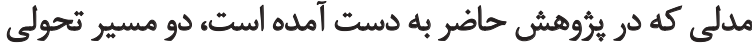
اختلال شخصيث مرزى رانشان مى دهند.

يروهش حاضر از دو نظر محدوديت داشت: اول اينكه،

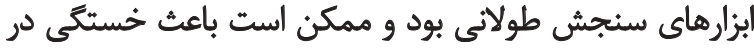

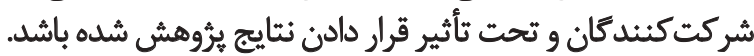

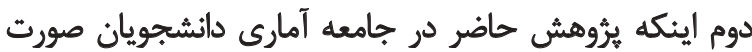

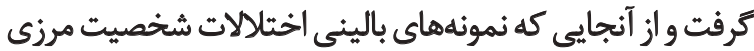

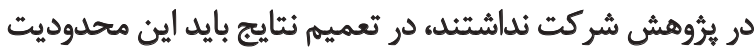

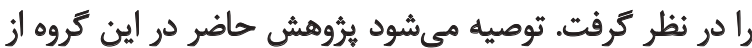

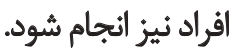

\section{سياستّز الوى}

بدين وسيله از جناب آقاى دكتر محسن مرادي، به دليل

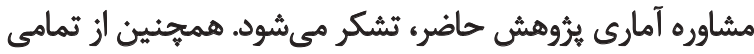

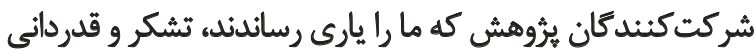

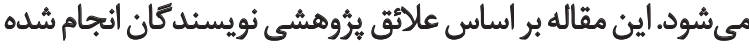
است و هيج سازمان و مؤسسهائ از آن حمايت مالى مالى نكرده است. بنا بر اظهار نويسنده مسئول مقاله، بين نويسندكان مقاله، تعارضى از نظر منافع وجود ندارد.
معتقدند بدرفتارى در دوران كودكىي، ممكن است باعث تداخل

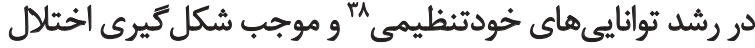

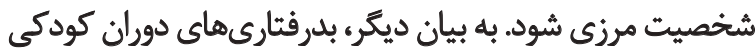

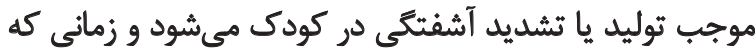

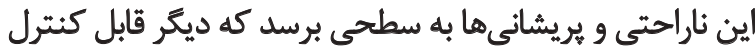

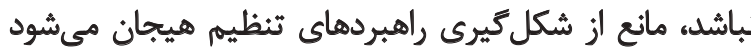

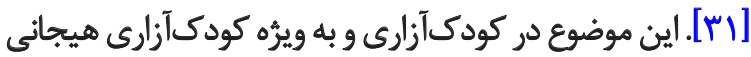

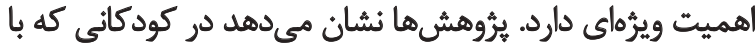

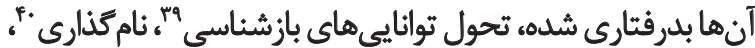

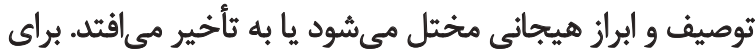

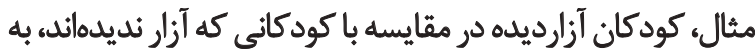

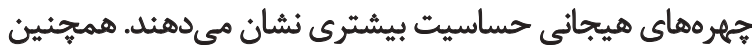

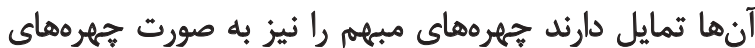

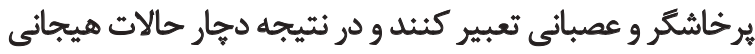

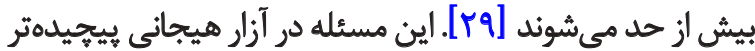

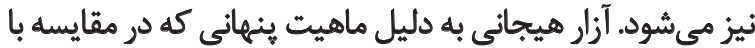

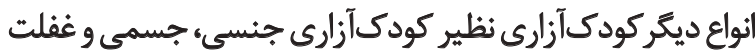

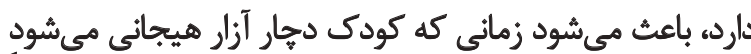

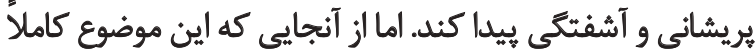

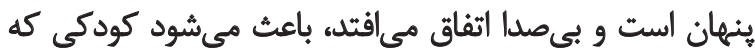

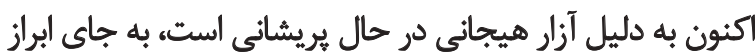

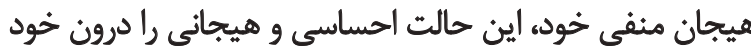

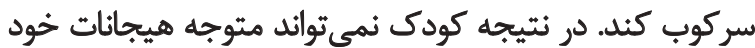

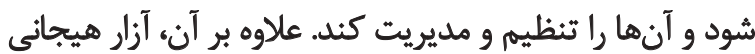

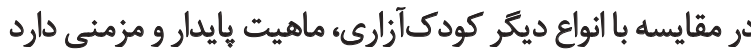

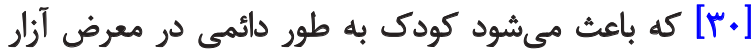

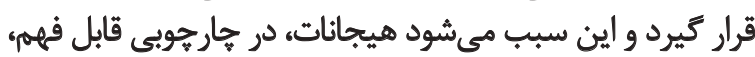

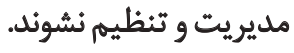

يُروهشها نشان داده است مؤلفههاى مربوط به تنظيمنشدن هيجانى، باميزان صفات اختلال شخصيت مرزى، در ارتبان ارتباط است.

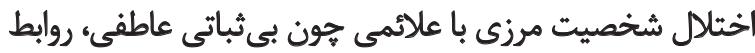

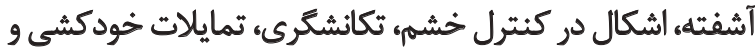

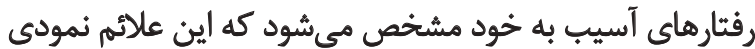

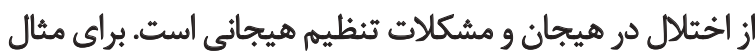

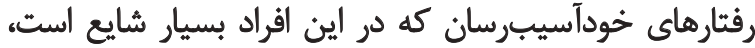

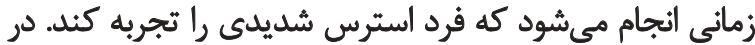

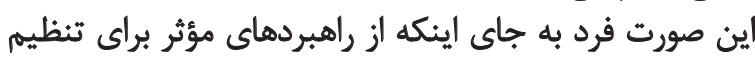

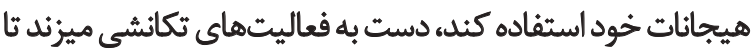

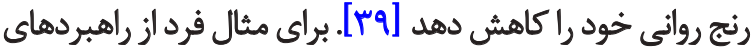

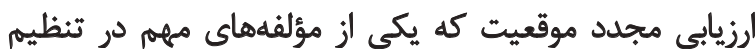




\section{References}

[1] Kaplan BJ. Kaplan and Sadock's Synopsis of Psychiatry: Behavioral Sciences/Clinical Psychiatry. Philadelphia, Pennsylvania: Lippincott Williams \& Wilkins; 2016.

[2] Pompili M, Girardi P, Ruberto A, Tatarelli R. Suicide in borderline personality disorder: A meta-analysis. Nordic Journal of Psy-chiatry. 2005; 59(5):319-24. [DOI:10.1080/08039480500320025] [PMID]

[3] Apfelbaum S, Regalado P, Herman L, Teitelbaum J, Gagliesi P. Comorbidity between bipolar disorder and cluster B personality disorders as indicator of affective dysregulation and clinical severity. Actas Espanolas de Psiquiatria. 2013; 41(5):269-78. [PMID]

[4] Kuo JR, Khoury JE, Metcalfe R, Fitzpatrick S, Goodwill A. An examination of the relationship between childhood emotional abuse and borderline personality disorder features: The role of difficulties with emotion regulation. Child Abuse \& Neglect. 2015; 39:147-55. [DOI:10.1016/j.chiabu.2014.08.008] [PMID]

[5] Zanarini MC, Yong L, Frankenburg FR, Hennen J, Reich DB, Marino MF, et al. Severity of reported childhood sexual abuse and its relationship to severity of borderline psychopathology and psychosocial impairment among borderline inpatients. The Journal of Nervous and Mental Disease. 2002; 190(6):381-7. [DOI:10.1097/00005053-200206000-00006] [PMID]

[6] Alilou MM, Hashemi T, Beirami M, Bakhshipour A, Sharifi MA. [Investigating the relationship between childhood maltreatment, loss and initial separation and emotional dysregulation with borderline personality disorder (Persian)]. Journal of Psychology Achievements. 2014; 21(2):65-88.

[7] Laporte L, Paris J, Guttman H, Russell J, Correa JA. Using a sibling design to compare childhood adversities in female patients with BPD and their sisters. Child Maltreatment. 2012; 17(4):31829. [DOI:10.1177/1077559512461173] [PMID]

[8] Trickett PK, Mennen FE, Kim K, Sang J. Emotional abuse in a sample of multiply maltreated, urban young adolescents: Issues of definition and identification. Child Abuse \& Neglect. 2009; 33(1):27-35. [DOI:10.1016/j.chiabu.2008.12.003] [PMID] [PMCID]

[9] Van Dijke A, Ford JD, van Son M, Frank L, van der Hart O. Association of childhood-trauma-by-primary caregiver and affect dysregulation with borderline personality disorder symptoms in adulthood. Psychological Trauma: Theory, Research, Practice, and Policy. 2013; 5(3):217-24. [DOI:10.1037/a0027256]

[10] Lieb K, Zanarini MC, Schmahl C, Linehan MM, Bohus M. Borderline personality disorder. The Lancet. 2004; 364(9432):453-61. [DOI:10.1016/S0140-6736(04)16770-6]

[11] Cassidy J, Phillip R. Handbook of attachment: Theory, research, and clinical application. New York: Guilford Press, 2016.

[12] Levy KN. The implications of attachment theory and research for understanding borderline personality disorder. Development and Psychopathology. 2005; 17(4):959-86. [DOI:10.1017/ S0954579405050455] [PMID]

[13] Aaronson CJ, Bender DS, Skodol AE, Gunderson JG. Comparison of attachment styles in borderline personality disorder and obses-sive-compulsive personality disorder. Psychiatric Quarterly. 2006; 77(1):69-80. [DOI:10.1007/s11126-006-7962-x] [PMID]

[14] Levy KN, Johnson BN, Clouthier TL, Scala J, Temes CM. An attachment theoretical framework for personality disorders. Ca- nadian Psychology/Psychologie Canadienne. 2015; 56(2):197-207. [DOI:10.1037/cap0000025]

[15] Gratz KL, Roemer L. Multidimensional assessment of emotion regulation and dysregulation: Development, factor structure, and ini-tial validation of the difficulties in emotion regulation scale. Journal of Psychopathology and Behavioral Assessment. 2004; 26(1):41-54. [DOI:10.1023/B:JOBA.0000007455.08539.94]

[16] Southam-Gerow MA, Kendall PC. Emotion regulation and understanding: Implications for child psychopathology and therapy. Clinical Psychology Review. 2002; 22(2):189-222. [DOI:10.1016/ S0272-7358(01)00087-3]

[17] Burns EE, Jackson JL, Harding HG. Child maltreatment, emotion regulation, and posttraumatic stress: The impact of emotional abuse. Journal of Aggression, Maltreatment \& Trauma. 2010; 19(8):801-19. [DOI:10.1080/10926771.2010.522947]

[18] IBM. IBM SPSS statistics for windows, version 22.0. Armonk, New York: IBM; 2013.

[19] Jöreskog KG, Sörbom D, du Toit S. LISREL 8: New statistical features. Portland: Scientific Software International; 2001. [PMID]

[20] Arbuckle JL. IBM SPSS Amos 19 user's guide. Crawfordville, Florida: Amos Development Corporation; 2010.

[21] Kline R. Principles and practice of structural equation modeling. New York: Guilford Press; 2015.

[22] Bernstein DP, Stein JA, Newcomb MD, Walker E, Pogge D, Ahluvalia T, et al. Development and validation of a brief screening ver-sion of the Childhood Trauma Questionnaire. Child Abuse \& Neglect. 2003; 27(2):169-90. [DOI:10.1016/S0145-2134(02)00541-0]

[23] Mikaeili N, Zamanloo K. [A study of the prevalence of child abuse and its prediction from parents' depression and anxiety, attach-ment styles and mental health of their adolescent boys (Persian)]. Psychology of Exceptional Individuals. 2012; 2(5):145-66

[24] Mohammadzadeh A. [Validation of the borderline personality inventory in Iran (Persian)]. International Journal of Behavioral Scienc-es. 2009; 5(3):13-24.

[25] Narimani M, Abbasi M, Abolghasemi A, Ahadi B. [A study comparing the effectiveness of acceptance/ commitment by emotional regulation training on adjustment in students with dyscalculia (Persian)]. Learning Disabilities. 2013; 2(4):154-76.

[26] Pakdaman S. [A Study on the Relationship between Attachment Styles and Socialization in Adolescence (Persian)] [PhD thesis]. Tehran: University of Tehran; 2003.

[27] Chou CP, Bentler PM. Estimates and tests in structural equation modeling. In: Hoyle RH, editor. Structural Equation Modeling: Concepts, Issues, and Applications. Thousand Oaks, California: SAGE; 1995.

[28] Hu L, Bentler PM. Cutoff criteria for fit indexes in covariance structure analysis: Conventional criteria versus new alternatives. Struc-tural Equation Modeling: A Multidisciplinary Journal. 1999; 6(1):1-55. [DOI:10.1080/10705519909540118]

[29] Westbrook J, Berenbaum H. Emotional awareness moderates the relationship between childhood abuse and borderline personality disorder symptom factors. Journal of Clinical Psychology. 2017; 73(7):910-21. [DOI:10.1002/jclp.22389] [PMID] 
[30] Iwaniec D. The emotionally abused and neglected child: Identification, assessment and intervention: A practice handbook. Hoboken, New Jersey: John Wiley \& Sons; 2006.

[31] Kim J, Cicchetti D. Longitudinal pathways linking child maltreatment, emotion regulation, peer relations, and psychopathology. Journal of Child Psychology and Psychiatry. 2010; 51(6):70616. [DOI:10.1111/j.1469-7610.2009.02202.x] [PMID] [PMCID]

[32] Winsper C, Hall J, Strauss VY, Wolke D. Aetiological pathways to Borderline Personality Disorder symptoms in early adolescence: childhood dysregulated behaviour, maladaptive parenting and bully victimisation. Borderline Personality Disorder and Emotion Dysregulation. 2017; 4(1):10. [DOI:10.1186/s40479-0170060-x] [PMID] [PMCID]

[33] Neumann E. Emotional abuse in childhood and attachment anxiety in adult romantic relationships as predictors of personality dis-orders. Journal of Aggression, Maltreatment \& Trauma. 2017; 26(4):430-43. [DOI:10.1080/10926771.2017.1299826]

[34] Critchfield KL, Levy KN, Clarkin JF, Kernberg OF. The relational context of aggression in borderline personality disorder: Using adult attachment style to predict forms of hostility. Journal of Clinical Psychology. 2008; 64(1):67-82. [DOI:10.1002/ jclp.20434] [PMID]

[35] Aghayousefi A, Saravani S, Zeraatee R, Razeghi FS, Pourabdol S. [Prediction of students' academic performance based on attachment styles and different levels of adjustment (Persian)]. Iranian Journal of Psychiatry and Clinical Psychology. 2016; 21(4):308-16.

[36] Hazan C, Shaver P. Romantic love conceptualized as an attachment process. Journal of Personality and Social Psychology. 1987; 52(3):511. [DOI:10.1037/0022-3514.52.3.511] [PMID]

[37] Maughan A, Cicchetti D. Impact of child maltreatment and interadult violence on children's emotion regulation abilities and socio-emotional adjustment. Child Development. 2002; 73(5):1525-42. [DOI:10.1111/1467-8624.00488] [PMID]

[38] Cicchetti D, Lynch M. Toward an ecological/transactional model of community violence and child maltreatment: Consequences for children's development. Psychiatry. 1993; 56(1):96118. [DOI:10.1080/00332747.1993.11024624] [PMID]

[39] Conklin CZ, Bradley R, Westen D. Affect regulation in borderline personality disorder. The Journal of Nervous and Mental Disease. 2006; 194(2):69-77. [DOI:10.1097/01. nmd.0000198138.41709.4f] [PMID]

[40] Bierer LM, Yehuda R, Schmeidler J, Mitropoulou V, New AS, Silverman JM, et al. Abuse and neglect in childhood: Relationship to per-sonality disorder diagnoses. CNS Spectrums. 2003; 8(10):737-54. [DOI:10.1017/S1092852900019118] [PMID]

[41] Kuo JR, Linehan MM. Disentangling emotion processes in borderline personality disorder: Physiological and self-reported assessment of biological vulnerability, baseline intensity, and reactivity to emotionally evocative stimuli. Journal of Abnormal Psychology. 2009; 118(3):531-44. [DOI:10.1037/a0016392] [PMID] [PMCID]

[42] Kuo JR, Fitzpatrick S, Metcalfe RK, McMain S. A multi-method laboratory investigation of emotional reactivity and emotion regulation abilities in borderline personality disorder. Journal of Behavior Therapy and Experimental Psychiatry. 2016; 50:52-60. [DOI:10.1016/j.jbtep.2015.05.002] [PMID] 
